\title{
Prevalence and Pattern of Allergic and Non Allergic Rhinitis in Hospitalised Patients in A Tertiary Health Care: A Cross Sectional Study
}

\author{
Md.nawad azam ${ }^{1}$, Modi Narayan Prasad ${ }^{2}$, Mohanty Anil Kumar ${ }^{3}$ \\ ${ }^{1}$ Resident, ${ }^{2}$ Associate Professor, ${ }^{3}$ Professor, Dept of pediatrics, S.C.B. medical college, Cuttack, India.
}

\begin{abstract}
:
Background: Rhinitis is inflammation of nasal mucosa leading to rhinorrhea, nasal obstruction, sneezing, itching etc. Although considered trivial, it has major impact on quality of life. The objective is to study the prevalence of Allergic rhinitis and non Allergic rhinitis in hospitalized patients with special reference to non respiratory cases and the association of perinatal events with Allergic rhinitis if any.

Material And Method: An observational study was conducted among patients (aged 1 year to 14 years) admitted to S.C.B. medical college during study period of January 2014-June 2014. Patients were enquired as per a predesigned questionnaire as per Allergic rhinitis and its impact on asthma (ARIA) guideline and were investigated to establish Allergic etiology.

Results: Among 472 in-patient studied, 17\% were found to have Allergic rhinitis (AR), 18\% had Non-Allergic rhinitis (NAR). Urban to rural prevalence for AR and NAR are 2:1 and 1.1:1(p-value 0.047) respectively. There is high prevalence of AR in non respiratory(15\%) as well as respiratory cases(23\%).Allergic rhinitis is 2.5 times more common in babies delivered by LSCS than VD ( $p$-value $=0.007,2.5: 1)$. Runny nose is the most common complaint among Allergic rhinitis where as nasal obstruction is the commonest in non-Allergic rhinitis. 48\% Allergic rhinitis were SPT positive, 16\% allergic rhinitis showed serum IgE above 200IU/L.

Conclusion: There is a high prevalence of Allergic and non-Allergic rhinitis in our country which go unnoticed evident from this study. Rhinitis, both Allergic and non-Allergic is also very common even in hospitalized patients for non respiratory complaints.
\end{abstract}

Key words: Allergic rhinitis/non Allergic rhinitis/ ARIA/ SPT/Serum IgE

\section{Introduction:}

Rhinitis is a common problem but definite data about its prevalence among pediatrics age group is still awaited. In adults its effect on social life, loss of work and health is well known but in children it has been trivialized by parents and physicians as common cold. Previous studies dealing with Allergic Rhinitis used questionnaires which overlooked many data and missed many cases.

According to International consensus report on diagnosis and management of rhinitis, two or more of nasal symptoms like rhinorrhea, itching/sneezing, congestion, anosmia for greater than $1 \mathrm{hr}$ a day suggests rhinitis $^{[1,4]}$. Allergic rhinitis is defined by Allergic sensitization leading runny nose, nasal congestion, itching or conjunctivitis in last 12 mo without associated viral 'flu'. This is supported by lab test like skin prick test (SPT) for sensitive allergen, Radioallergosorbent test (RAST), serum IgE. But there is no specific criterion for diagnosis of non Allergic rhinitis hence it is considered in pts with persistent rhinitis symptoms without allergy $[2,3]$.

Although many studies on rhinitis are conducted in adults but pediatric case studies are limited. Mean age for Allergic rhinitis is considered 14-16 yrs. Yet it is very well documented in pediatrics age groups ${ }^{[6]}$. Many genetic and environmental conditions are related to rhinitis, and there associations to pediatric cases are yet to be probed.

Our study had included pediatric patients of varied age with different socio-economic status, and there associations with different perinatal events were analyzed.

\section{Material And Methods:}

An observational study was conducted among patients admitted to S.C.B. medical college during study period of January 2014-June 2014. All the pediatric patients beyond 1yr and below 14 yrs age were included in the study. Patients with pneumonia, asthma, Bronchiolitis, upper respiratory tract infection were included as respiratory cases and those patients with no respiratory ailment were grouped under Non-respiratory cases. Patients were screened for allergic rhinitis (AR) and Non allergic rhinitis and those patients with allergic rhinitis were further investigated for birth weight, mode of delivery, gestational age and NICU admission. Parents were 
enquired as per a predesigned questionnaire (table-1) about symptoms pertaining to rhinitis. The questionnaire is prepared as per the GINA guideline and ARIA protocol.

The questions asked whether the patient have any one or more of the symptoms like watery runny nose, sneezing (in bouts), nasal obstruction, nasal itching, and loss of smell for at least one hour on most days or on most days in any season suggesting Allergic rhinitis. Similarly a set of questions including whether patient have unilateral symptoms, thick yellow discharge from nose, postnasal drip, recurrent nasal bleed etc helps in excluding allergic rhinitis and considered in non allergic rhinitis.

All were asked about symptoms of rhinitis and those with repeated episodes were further enquired about family history of allergy, exposure to allergens and seasonal variations. Patients with rhinitis underwent physical examination to exclude sinusitis, DNS, viral proderm and other infectious causes. Parents were asked about the socio-economic status and medical history for above symptoms.

Following a detail history and clinical examination, patients with signs and symptoms of rhinitis were subjected to Allergic testing like skin prick test (SPT) to common allergen and total serum IgE estimation as described before .Patients with SPT positive and/ or total serum IgE > 200IU/L with clinical features of rhinitis were grouped under Allergic rhinitis and rest of the rhinitis were considered of non Allergic etiology.

Skin prick test (SPT) for allergen is classic method to verify atopy. In this method a drop of test solution of usual allergens like pollen, dander, dust mite, moulds and foods etc. is placed over fore- arm and skin is pricked through the drop with the help of a sharp needle. Following which immediate reactions like wheal or flare is observed within 15 mins. The test value is then compared with positive (Histamine) and negative (Normal Saline) control. Total Serum IgE is estimated by enzyme linked immunsorbent assay.

\subsection{Perinatal Variables:-}

Patients with Allergic rhinitis according to clinical criteria were inquired about perinatal events like gestational age, weight, mode of delivery, NICU admission in neonatal period.

\subsection{Statistical Analysis:-}

All the data were compiled and analyzed. Statistics was applied on data for analysis and tabulation using SPSS software. Bivariate analysis was performed using fisher's exact t-test and p-value is calculated for perinatal variables.

\section{Results:-}

Of the 472 in- patients studied, 35\% pts had rhinitis and among them 17\% pts had Allergic and 18\% pts had non Allergic rhinitis. In Allergic rhinitis female prevalence (18\%) was found to be more than male (16\%). On the other hand for NAR male prevalence (19\%) was higher than female (16\%). (Fig-1)

Mean age of the study sample is 6 yrs 6 months. Age distribution shows a higher affection of older age groups in both Allergic and non Allergic rhinitis (Fig-2). 392 out of the 472 patients presented with non respiratory complaints Prevalence of both AR and NAR in non-respiratory cases is $15 \%$ and $18 \%$ respectively and for respiratory cases it is $23 \%$ and $29 \%$ respectively (table-2). Out of the 472 cases 224 belong to lower middle socio-economic status. Prevalence of Allergic rhinitis in this group is 15\%. Major proportions of cases in our study belong to rural area (80\%) than urban (20\%). But urban prevalence of Allergic rhinitis (20\%) was found to be high. Comparing RR of AR to NAR in urban to rural, there was more prevalence of Allergic rhinitis $(2: 1)(\mathrm{p}$-value $=0.047)$ in urban area which is significant. Parental history of atopy of at least one parent was found in $24.6 \%$ of Allergic rhinitis.

For variables of perinatal events we calculated the odds ratio (OR) by taking cases as those who meet criteria for Allergic rhinitis and control who didn't meet the criteria but of same age group range. Among Allergic rhinitis $2.5 \%$ were preterm and $97.5 \%$ were term at birth. We found an OR of 0.21 with p-value 0.058 which was statistically not significant for relationship of AR with gestational age $<37$ wks (preterm). For mode of delivery we found $\mathrm{OR}=3$ for relationship of AR with LSCS and p-value being 0.007 which highly significant (Table-3). We found no significant association of gestational weight with AR as OR for the same was 0.84(pvalue 0.69 ). Analyzing the NICU admission in neonatal period OR was found to be1.7 and p-value 0.32 . The above result is statistically insignificant. Allergic rhinitis presented with clear runny nose (71\%) followed by sneezing (67\%), whereas most common presentation for non Allergic rhinitis was nasal obstruction (49.4\%), (Table-4).

Mean total serum IgE level in Allergic rhinitis group was $260 \mathrm{IU} / \mathrm{L} .38$ out of 80 (48\%) Allergic rhinitis were only SPT positive, 26(32\%) showed IgE > 200 IU/L as well SPT positivity, where as $16(20 \%)$ showed only serum IgE above $200 \mathrm{IU} / \mathrm{l}$. Median eosinophil count was $6 \%$ and median absolute eosinophil count was $354 / \mathrm{mm} 3$. 


\section{Figures And Tables:}

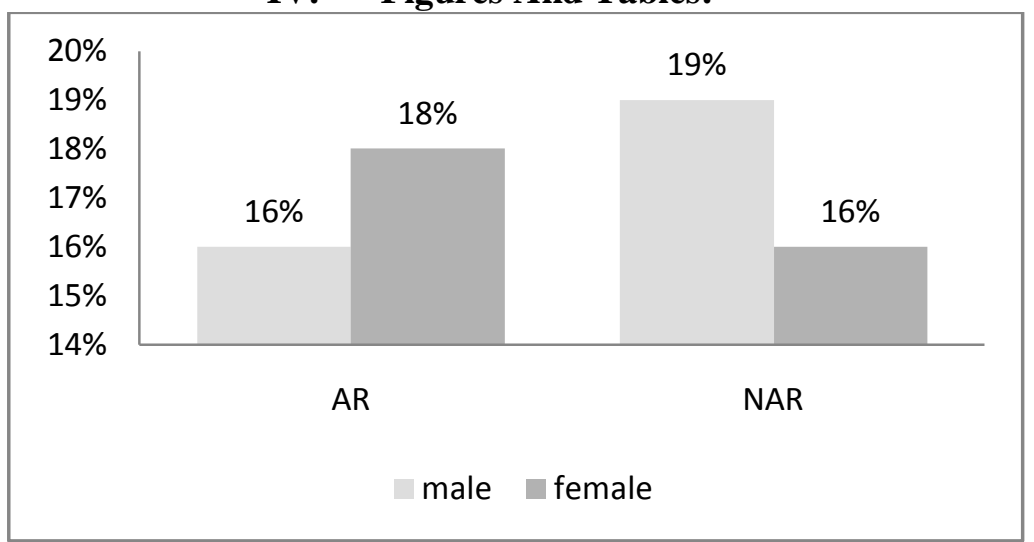

Fig: 1 Gender Prevalence of Allergic and Non-allergic rhinitis

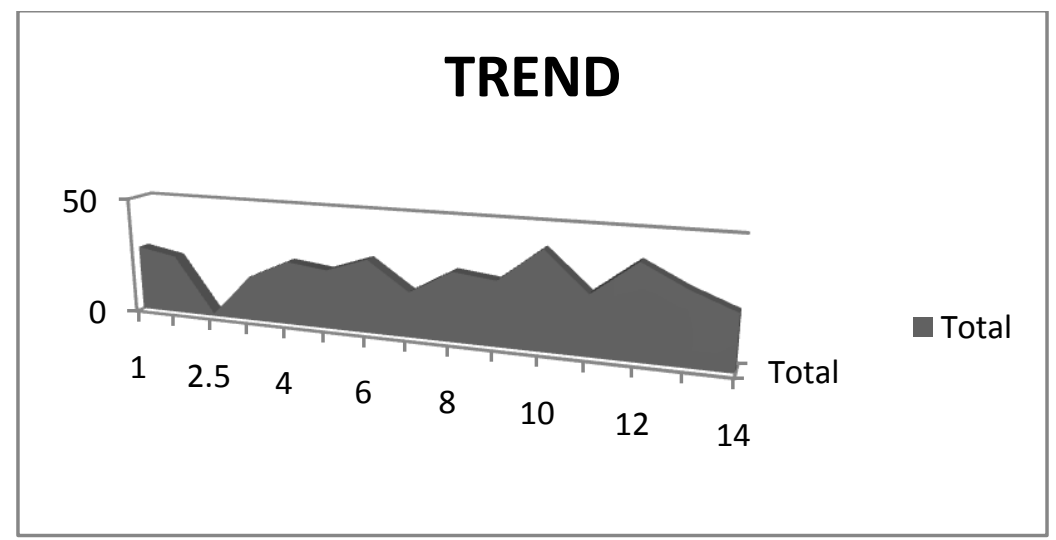

Fig: 2 Age trend in Allergic rhinitis

TABLE -1

\begin{tabular}{|l|l|l|}
\hline Allergic Rhinitis questionnaire & & \\
\hline $\begin{array}{l}\text { Do u have any of the following symptoms for at least on hour on most days (or on most } \\
\text { days during any season)? }\end{array}$ & & \\
\hline Watery runny nose & Yes & No \\
\hline Sneezing especially violent and in bouts & Yes & No \\
\hline Nasal obstruction & Yes & No \\
\hline Nasal itching & Yes & No \\
\hline Conjunctivitis(red, itchy eyes) & Yes & No \\
\hline Do u have any of the following symptoms? & & \\
\hline Symptoms on one side of your nose & Yes & No \\
\hline Thick, green or yellow discharge from your nose & Yes & No \\
\hline Postnasal drip & Yes & No \\
\hline Facial pain & Yes & No \\
\hline Recurrent nosebleeds & Yes & No \\
\hline
\end{tabular}

Note : the symptoms of question 2 is usually not found in allergic rhinitis. The presence of any one of them suggests that alternate diagnosis should be investigated.

TABLE-2 Prevalence AR and NAR in Non-respiratory and Respiratory cases

\begin{tabular}{|l|l|l|}
\hline & Allergic rhinitis & Non allergic rhinitis \\
\hline Non-respiratory case & $15 \%$ & $18 \%$ \\
\hline Respiratory case & $23 \%$ & $29 \%$ \\
\hline
\end{tabular}

TABLE-3 Relationship of allergic rhinitis with mode of delivery:

\begin{tabular}{llll}
\hline Variables & AR(\%) & NAR(\%) & Total \\
\hline LSCS & $18(22 \%)$ & $6(7 \%)$ & 24 \\
VD & $62(78 \%)$ & $79(93 \%)$ & 141 \\
Total & 80 & 85 & 165 \\
\hline
\end{tabular}

$\mathrm{OR}=3 \quad \mathrm{p}$-value $=0.007$ 


\begin{tabular}{|lll|}
\hline \multicolumn{2}{|l|}{ Table-4 Presenting Symptoms In Rhinitis } & \\
\hline Variables & Allergic rhinitis(n=80).no.(\%) & Non allergic rhinitis(n=85).no.(\%) \\
\hline Runny nose & $71(88 \%)$ & $1(1.1 \%)$ \\
Sneezing & $54(67 \%)$ & $1(1.1 \%)$ \\
Conjunctivitis & $21(26 \%)$ & $1(1.1 \%)$ \\
Nasal obstruction & $51(67.7 \%)$ & $42(49.4 \%)$ \\
Unilateral symptom & 0 & $8(9.4 \%)$ \\
Mucopurulent rhinorrhea & $2(2.5 \%)$ & $38(44.7 \%)$ \\
\hline
\end{tabular}

\section{Discussion And Conclusion:}

Rhinitis is a common problem in children which goes unnoticed in childhood. There are only a few studies regarding this problem in pediatric age group. Patients admitted to our hospital irrespective of respiratory or non respiratory ailments were searched for rhinitis and its association.

There was no clear criteria for diagnosis of allergic and non Allergic rhinitis but recently an evidence based guideline for Allergic rhinitis, developed by international experts in

association with the World Health Organization (WHO) $)^{[2,5]}$, which is helping health professionals reduce the considerable morbidity and disability related to this condition. Prevalence of NAR is still unknown in general population. In our study prevalence of NAR (18\%) is more than AR (17\%). In our study female prevalence $(18 \%)$ of Allergic rhinitis is more than male $(16 \%)$. Although many study showed increased prevalence of allergic rhinitis in female after adolescent age ${ }^{[7]}$. There is an increasing trend of prevalence with age in our study as more cases are reported in 11 to 14 yrs of age . This is in accordance with previous reports suggesting a peak at 13 to 14 yrs. Urban prevalence of Allergic rhinitis was significantly high (20\%). Similar high prevalence was found in a study conducted in Poland and other studies ${ }^{[7,8]}$.

Prevalence of Allergic rhinitis in patients who presented with non respiratory complaint was $15 \%$ and in those with respiratory ailments was $23 \%$. A population study in Bangladesh (ISAAC STUDY) has shown a prevalence of Allergic rhinitis in non respiratory cases $20 \%{ }^{[10]}$. Our study though conducted in a hospital setting has similar results. Presence of such high prevalence allergic rhinitis in non respiratory and respiratory cases suggests many cases remain undetected and unattended in our society. Family history of atopy was found in $24.6 \%$ ( OR=2.1) of patients with Allergic rhinitis.

Among perinatal variables only mode of delivery showed a significant association with later Allergic rhinitis. Odds ratio of association of LSCS with AR was 3(p-value=0.007). Similar association was also found with 3 fold increase in Allergic complaints in children born by caesarean section. (Eggesbo et al) and other studies ${ }^{[10,11]}$. But gestational age, birth weight, prior NICU admission did not show any significant relation with $\mathrm{AR}^{[12,13]}$.

Most common symptoms for Allergic rhinitis are runny nose followed by sneezing, where as NAR presents with nasal obstruction. This is in accordance with Bachert et al and Ryden et al study reports ${ }^{[1,14]}$. In a study conducted in 2011, highest IgE levels was found in patients with both asthma and eczema (985 IU/ml) followed by asthma alone (305 IU/ml), eczema alone (273IU/ml) and then Allergic Rhinitis $(171 \mathrm{IU} / \mathrm{ml})^{[15]}$.

Limitation of the study was sensitivity and specificity of both skin prick test as well as total serum IgE. It is still debatable as the results are affected by many disease condition and drug intake but these tests are valuable adjunct to the diagnosis of Allergic disease. Yet diagnosis and treatment of Allergic rhinitis still depends on a good clinical evaluation.

In conclusion we observe a higher prevalence of undetected rhinitis and Allergic rhinitis in odisha even in lower socioeconomic strata. With higher prevalence of allergic rhinitis in female children and in urban children.. Runny nose is the commonest presentation in AR where as NAR presents with nasal obstruction. Most of the perinatal events don't have any effect on later development of Allergic rhinitis except mode of delivery by caesarean section.

There is a high prevalence of allergic rhinitis in non respiratory hospitalized cases which indicates a lot of cases of allergic rhinitis remain un-detected in pediatric population which may have great impact on quality of life in adulthood. 


\section{References}

[1]. C. Bachert, Persistent rhinitis - allergic or nonallergic, Allergy 2004: 59 (Suppl. 76): 11-15

[2]. 2.Quillen David M, and Feller David B, Diagnosing Rhinitis: Allergic vs. Nonallergic, American Family Physician 2006;73: 158390

[3]. Blaiss MS. Allergic rhinoconjunctivitis: burden of disease. Allergy Asthma Proc. 2007;28:393-397

[4]. Skoner DP. Allergic rhinitis: definition, epidemiology, pathophysiology, detection, and diagnosis. J Allergy Clin Immunol. 2001;108(suppl):S2-S8

[5]. Bousquet J,Van-Cauwenberge P, Khaltaev N,and the ARIA group. Allergic Rhinitis and Its Impact on Asthma. J Allergy Clin Immunol 2001;108(suppl):S147-S333

[6]. The International Study of Asthma and Allergies in Childhood (ISAAC) Steering Committee. Worldwide variation in prevalence of symptoms of asthma, Allergic Rhino-conjunctivitis, and atopic eczema: ISAAC. Lancet 1998;351:1225-32.

[7]. Osman M, Hansell A.L,Simpson C.R,Hollowell J,Helms P.J,Gender specific presentation of asthma, Allergic rhinitis, eczema in primary care, Primary care respiratory journal.2007;16:28-35

[8]. Izabela Kupryś-Lipińska, Anna Elgalal, Piotr Kuna, Urban-rural differences in the prevalence of atopic diseases in the general population in Lodz Province (Poland), Post Dermatol Alergol 2009; XXVI, 5: 249-256

[9]. Jessen M, Janzon L. Prevalence of nonAllergic nasal complaints in urban and rural population in Sweden. Allergy 1989;44:582587

[10]. Chowdhury M.A, Kabir ARM Lutful, Md. Monjurul Alam,Allergic rhinitis, asthma and atopic diseases: Bangladesh perspective, Bangladesh J of Otorhinolaryngology 2008; 14(2) : 66-70

[11]. Eggesbo M, Botten G, Stigum H, et al. Is delivery by cesarean section a risk factor for food allergy, J Allergy Clin Immunol 2003;112:420-6.

[12]. Michael Pistiner, Diane R, MPH,b,e Hassen Abdulkerim, Elaine Hoffman,Juan C.,Celedó n, Birth by cesarean section, Allergic rhinitis, and Allergic sensitization among children with a parental history of atopy, J Allergy Clin Immunol 2008;122:274-9.

[13]. Nafstad P, Magnus P, Jaakkola JJ. Risk of childhood asthma and Allergic rhinitis in relation to pregnancy complications. J Allergy Clin Immunol 2000;106:867-73

[14]. Annesi-Maesano I, Moreau D, Strachan D. In utero and perinatal complications preceding asthma. Allergy 2001;56:491-7.

[15]. Demirjian M., Rumbyrt J.S, Gowda V.C., Klaustermeyer W.B.,Serum IgE and eosinophil count in Allergic rhinitis---Analysis using a modified Bayes' theorem, Allergol Immunopathol (Madr). 2012;40(5):281-287

[16]. Ryden O, Andersson B, Andersson M, Disease perception and social behaviour in persistent rhinitis: a comparison between patients with Allergic and nonAllergic rhinitis, Allergy2004; 59: 461-464

[17]. Akbari Hedaiat, Hosseini R.F, Miri S, Amin R,The Prevalence of Allergic Rhinitis among 11-15 Years-old Children in Shiraz, IJI.2004;1:133-137

[18]. Siegel CJ, Dockhorn RJ. An evaluation of childhood rhinorrhea. Ann Allergy 1982; 48(1):9-11.

[19]. Howarth PH, von Mutius E, Martinez FD. Allergic and non Allergic rhinitis. Natural history, development and prevention of Allergic disease in childhood. In:Middleton's allergy principles and practice.2003;1:391-407

[20]. Bauchau V, Durham S.R, Prevalence and rate of diagnosis of Allergic rhinitis in Europe, Eur Respir J 2004; 24: 758-764

[21]. Leynaert B, Neukirch C, Liard R, Bousquet J, Neukirch F, Quality of life in Allergic rhinitis and asthma. A population based study of young adults. Am J Respir Crit Care Med 2000; 162: 1391-1396.

[22]. Andersson M, Greiff L, Svensson C, Persson CGA. Allergic and non-Allergicrhinitis. In: Busse WW, Holgate ST, editors. Asthma and rhinitis, 2nd edn. Oxford: Blackwell Science Ltd, 2000:232-244.

[23]. Bener A,Janahi I, Association between Childhood Atopic Disease and Parental Atopic Disease in a Population with High Consanguinity, Coll. Antropol. 29 (2005) 2: 677-682 